\title{
Massive stars in galaxies at high redshift: SMM J 14011+0252
}

\author{
Duilia F. de Mello, Tommy Wiklind \\ Onsala Space Observatory, \\ Chalmers Centre for Astrophysics and Space Science, \\ S-43992 Onsala, Sverige
}

\author{
Claus Leitherer \\ Space Telescope Science Institute, \\ 3700 San Martin Drive, Baltimore, MD21218, USA
}

\begin{abstract}
We present high resolution VLT spectra of the optical identification of the high-redshift galaxy SMM J14011+0252. We concentrate on the C IV $1550 \AA$ region of the spectra and compared the data with a spectral synthesis model generated with STARBURST 99 .
\end{abstract}

\section{Introduction}

As shown for the Lyman-break galaxy MS 1512-cB58 ( $z=2.73$ ), good signal-tonoise UV spectra of high- $z$ galaxies can provide a comprehensive study of star formation at early times (Leitherer et al. 2001; de Mello et al. 2000; Pettini et al. 2000). The stellar photospheric lines are diagnostics of the stellar population of the galaxy, whereas the interstellar lines probe the kinematics of the gas. The stellar wind lines are radiatively driven and can be used to estimate qualitatively the metallicity. Many of these lines are, however, weak and contaminated by interstellar components requiring high signal-to-noise and high-resolution data. In this contribution we present a high-resolution spectrum of SMM J 14011+0252 taken with the Very Large Telescope (VLT-Fors2). This source is one of the only two high- $z$ sources detected with the JCMT submm Common-User Bolometer Array (SCUBA) with secure optical identification. It is also the only one which shows no sign of AGN activity (Ivison et al. 2001 and references therein). Two objects called J1 and J2 near the SCUBA coordinates have been identified. A HST (F702W) image of SMM J 14011+0252 shows a complex system around these objects. Based on the offset between the HST coordinates and the CO and radio maps, it has been suggested that $\mathrm{J} 1$ and $\mathrm{J} 2$ are actually part of just one large galaxy obscured by dust. We present the high-resolution spectroscopy used to analyse the stellar content, the metallicity and dynamics of J1 and J2.

\section{Analysis and conclusions}

We used the upgraded STARBURST 99 package to calculate the synthetic UV spectra (Leitherer et al. 2001). We refer to http://www.stsci.edu/science/starburst99 

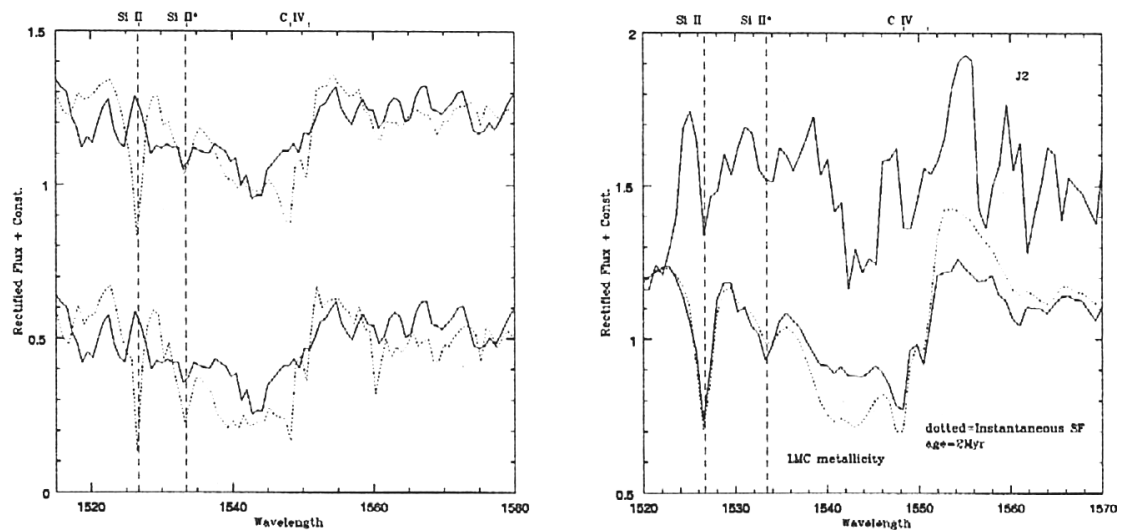

Figure 1. Left: Comparison between the observed spectrum of SMM J $14011+0252 \mathrm{~J} 1$ in the region of C IV $1550 \AA$ with the STARBURST 99 model (dotted line). Salpeter IMF, continuous star formation, $100 \mathrm{Myr}$ old. Left bottom: solar metallicity; Left top: LMC metallicity. Right bottom: Continuous line: Salpeter IMF, LMC metallicity, continuous star formation, and $100 \mathrm{Myr}$ old. Dotted line: Salpeter IMF, LMC metallicity, instantaneous star formation and $2 \mathrm{Myr}$ old; Right top: spectrum of SMM J 14011+0252 J2. Si II $1526.70,1533.44 \AA$ lines marked with vertical dashed lines; spectra rectified.

for more details on each individual parameters such as the IMF, and the star formation mode (continuous or instantaneous). The upgraded version includes a library of stars from the LMC. We concentrate on a comparison of the CIV $1550 \AA$ region of spectrum and the set of models compiled with STARBURST99. Figure 1 shows the synthetic models and the spectra of J1 and J2. Our main conclusions are: (i) the strength of the Si II $1533.44 \AA$ photospheric line and the shape of CIV $1550 \AA$ absorption component is better reproduced by the LMC model with continuous star formation and age $t=100 \mathrm{Myr}$. However, the shape of the profile is still very different from the model. This difference is probably due to a lower metallicity than in the LMC for $\mathrm{J} 1$; (ii) the Si II $1533.44 \AA$ photospheric line was used to obtain the redshift of $\mathrm{J} 2(z=2.5643)$. J1 is at $z=2.5650$, which translates into $\Delta v=200 \mathrm{~km} \mathrm{~s}^{-1}$; (iii) the strong P-Cygni profile in J2 suggests the presence of a young population in this region (or a weak AGN) and is better reproduced by a 2 Myr old single burst model; and (iv) the resonance line Si II $1526.70 \AA$, which has a narrow and deep interstellar component, is blue-shifted in J1 by $\Delta v \simeq 466 \mathrm{~km} \mathrm{~s}^{-1}$, typical of large scale outflows. These results are in agreement with the suggestion that these two objects are regions of star formation in one single galaxy. However, three separate units (J1, J2 and the dust source) cannot be ruled out at the present stage.

\section{References}

de Mello, D.F., Leitherer, C., Heckman, T.M. 2000, ApJ 530, 251

Ivison, R.J., Smail, I., Frayer, D.T., et al. 2001, ApJ (Letters) 561, L45

Leitherer, C., Leão, J.R.S., Heckman, T.M., et al. 2001, ApJ 550, 724

Pettini, M., Steidel, C.C., Adelberger, K.L., et al. 2000, ApJ 528, 96 